\title{
Encoding and retrieval effects of dual sensory-semantic cues
}

\author{
DOUGLAS L. NELSON and RICHARD C. BORDEN \\ University of South Florida, Tampa, Florida 33620
}

\begin{abstract}
This experiment was designed to explore the relative effects of dual and embedded sensory-semantic retrieval cues (e.g., GLAMOUR GIRL). Cues and targets shared both first-letter and associative features, only first-letter features, or only associative features. The cues were presented during the single test trial or they were presented during the single study trial and again at test. Finally, subjects in the dual-cue condition were or were not told about the addition of the first-letter contingency. The results indicated that dual were superior to single cues with each feature contributing independently and additively to recall. This dual facilitation was obtained when the cues were shown only at test or during both study and test and regardless of whether or not the subjects were explicitly aware of the additional sensory contingency. These findings were expected and explained by the assumptions of the coding-access-recognition model.
\end{abstract}

When given the word GLAMOUR and the instruction to write the first word that comes to mind, $24 \%$ of the respondents write GIRL. Similarly, given the word $D A I N T Y, 24 \%$ write GIRL. Finally, given the word GHOSTLY, no one writes GIRL. If the word GIRL is now encoded in a memory experiment, which cue will be more effective in aiding its recall, GLAMOUR, DAINTY, or GHOSTLY? Comparison of these cues indicates that they differ in number and in type of overlapping attributes with the target. GLAMOUR shares both a first letter and an associative relationship, $D A I N T Y$ only an equivalent associative relationship, and GHOSTLY only a first-letter relationship. The questions of interest concern the relative effectiveness of the dual cue. Will the dual sensory-associative cue be superior to the associative cue alone? To the sensory cue alone? If so, will this increased effectiveness be dependent upon specifically encoding this information during study or will the increased effectiveness also be apparent if the cue is presented only during testing, after the encoding has taken place? Must the subject be told about the addition of the sensory cue to the associative cue before the dual cues will be superior? And, finally, will the effect of compounding the cues be less than or greater than the additive effect of the individual cues?

The present experiment was designed to answer these questions. The results were expected to be relevant to conceptualizations of word encoding and retrieval, particularly those assuming that information corres-

This research was supported by Grant MH 16360 to the first author from the National Institute of Mental Health. Requests for reprints should be sent to Douglas L. Nelson, Department of Psychology, University of South Florida, Tampa, Florida 33620. ponding to both appearance and significance is encoded (e.g., Craik \& Tulving, 1975; Nelson, Wheeler, Borden, \& Brooks, 1974). For example, the coding-accessrecognition (CAR) model of Nelson et al. (1974) can be characterized by three assumptions. First, the coding of a word consists of activating representations that correspond to its visual, phonemic, and semantic features. The activation of visual and phonemic features typically precede the activation of meaning features but not through a stage-by-stage process. Encoding is assumed to be continuous, occurring through time with several independent types of features being processed at any given moment in time. Thus, word coding typically begins with at least minimal processing of appearance and, almost simultaneously, with the activation of significance. The word typically is emphasized because it seems reasonable to suppose that there are tasks in which meaning is anticipated in advance of sensory activation, a process that may occur while encoding redundant material such as meaningful text. Nevertheless, given that both sensory and meaning codes are activated, sensory codes are presumed to be less effective as memory mnemonics since they are less unique and differentiating. For a set of unrelated words, the sharing of visual features, of lines, curves, and angles, would be greater than the sharing of meaning features, of associative and semantic characteristics.

The second and third assumptions of the model attempt to specify retrieval processes. At the test for recall, the cue serves to provide access to information that has been encoded with it during the task or prior to the task. This access is presumed to be orderly in that the cue is likely to elicit well encoded information before poorly encoded information, or strong before weak items. Finally, according to the third assumption, as each piece of information or as each item is accessed, 
its features are sampled to determine if they were activated during the encoding phase of the task.

The CAR model combines assumptions of a "levels" of processing approach to word encoding with a variation of the generation-recognition approach to word retrieval (cf. Bahrick, 1970; Craik \& Lockhart, 1972). As such, the model leads to the expectation that dual sensory-associative cues should be superior to single cues. Since each type of cue independently facilitates recall, combining the cues should reflect their dual and additive influence (Nelson et al., 1974). However, the relative effectiveness of each type of cue should vary as a function of whether or not the cue is available during encoding. First-letter sensory cues should be least affected by this manipulation. Presenting cues that share an initial letter with their targets would not activate any unique additional information that is not already activated when the targets are presented alone. In fact, recall of the targets would be reduced to the extent that processing is focused upon appearance at the cost of activating semantic attributes (Craik \& Tulving, 1975). By contrast, associative cues would be most affected by presenting them during study, especially if the associative cues are relatively weak. The unique associative attributes of the target as it specifically. relates to the cue are more likely to be activated than when the target is presented alone. Therefore, if a cue provides access to its target during retrieval, recognition of the target as a list member should be enhanced.

\section{METHOD}

\section{Design}

The experimental conditions are illustrated in Table 1. Location of cue was combined with cuing conditions in a 2 by 5 between-subjects factorial. Hence, cues were located or presented only during test or during both study and test. In the dual-cue conditions, all cue-target pairs were associated and shared the same first letter (e.g., GLAMOUR GIRL). Subjects either were or were not informed about the first-letter sharing. All were told about the associative relationship. In the single-cue conditions, the cues were associatively related words (e.g., DAINTYGIRL) or they were the first letters of the targets. First-letter cues were either presented alone (e.g., $G$ GIRL) or embedded as the first letters of cue words that were associatively unrelated to their targets (e.g., GHOSTL Y GIRL). All subjects in the single-cue conditions were informed about the nature of the relationship between cues and targets. Finally, an additional control was used in conjunction with the studytest condition. Unrelated words served as cues during both study and test (e.g., ACCOUNT GIRL). A parallel free recall control for the test-only conditions was not included because it was felt that the processes involved in free and cued recall could potentially be very different, too different to allow meaningful comparison.

\section{Materials}

The 16-pair word lists were constructed with the aid of the Shapiro and Palermo (1965) norms. First, the dual-cue pairs were selected by taking stimuli whose primary responses shared the same initial letter. When these lists were completed, the single-cue, associatively related word lists were formed by selecting pairs that matched dual-cue pairs in associative strength. However, these pairs did not share first letters. Two different but presumably comparable lists were constructed for each condition. For the dual-cue condition, the lists were ATTENTION ARMY, BROWN BLACK, COMPACT CAR, DREARY DAY, FRAUD FAKE, GLAMOUR GIRL, HAIR HEAD, JELLY JAM, LESSON LEARN, MINOR MAJOR, NICEL $Y$ NEAT, PAPER PEN, RUGGED ROUGH, SIGN STOP, TRUNK TREE, WATER WET and ATTENTION ARMY, BALL BOUNCE, CRACKER CRUMB, DUNGEON DARK, FLUTTER FLY, GLITTER GOLD, HEAD HAIR, JUNE JULY, LIKE LOVE, MYSTIC MAGIC, NICEL Y NEAT, PARTICLE PIECE, ROSE RED, STROKE SWIM, TRAIN TRACK, WELL WATER. The single-cue, related word lists were $B L O W \mathrm{HARD}, C O R D I A L$ DRINK, DAINTY GIRL, FROSTY COLD, GALLON JUG, NOTE MUSIC, OATH SWEAR, LOG WOOD, HELP AID, $M A D E$ BUILD, VOICE LOUD, RUSTIC OLD, STOLEN THIEF, TENSE NERVOUS, ARTIST PAINT, WALL FLOOR and $B L A D E$ GRASS, CLUMSY AWKWARD, EXAMINE LOOK, FATAL DEATH, GENTLE KIND, HAT COAT, IDEAL PERFECT, NOTE MUSIC, MEAT STEAK, OBVIOUS EASY, PROSPER RICH, RUSTY NAIL, STOLEN THIEF, TRAIN WHISTLE, $A G I L E$ QUICK, WALL FLOOR. For each of these lists, the mean associative strength between pairs was the same, averaging .19 (with $\mathrm{SD}=.06$ ). The low level of strength was intentionally selected. Too high a level conceivably could have produced a ceiling effect so that the addition of the sensory cue might not have shown its effects.

The lists for the two associatively unrelated lists also were matched to the dual-cue conditions. The cues sharing initial letters were yoked to the targets listed in the above order and were ACCOUNT (for ARMY), BRIEF (for BLACK), CORDIAL, DESOLATE, FLUTE, GHOSTLY, HOME, JACKET, LAMP, MARGIN, NOTABLE, PEOPLE, RECENT, SOIL, TOWN, WORLD and ACCOUNT, BOOK, CLIENT, DAGGER, FLOURISH, GLIMPSE, HILL, JACK, LIFT, MINDFUL, NOTABLE, PAVILION, ROCK, SEARCH, TABLE, WOLF. To form the unrelated word study-test condition, the above cues were simply reassigned to other targets in the list (e.g., ACCOUNT was assigned to GIRL). In the first-letter-only condition, the cues were the first letters of the targets.

Thorndike and Lorge (1944) frequency was equated in all lists and, in addition, all extraneous sensory and semantic relationships within each list were minimized.

\section{Procedure}

All subjects participated in individual sessions. Words and letters were typed in uppercase letters and were presented with a Kodak Carousel slide projector for a single study-test trial. Items were shown for $3 \mathrm{sec}$ during study, and the presentation of cues during test was response paced. The next cue was shown as soon as the subject responded to the current cue. The order of presentation of all items was independently randomized on study and on test for every subject.

During study, all subjects were required to read the items aloud as they appeared and, at test, the cues also were read aloud prior to response anticipation. When cues were located on study and test, the subjects were told that words appearing on the right were targets and that, since they would only be shown once, it was important to concentrate and remember as many targets as possible. These subjects also were told that the underlined word (or letter) appearing on the left was a cue word, that the cue words would be presented during the test phase, and that concentrating on the relationship between the cue words and their targets during study would aid recall of targets during test. As already noted, subjects were fully informed about the nature of the relationship or relationships, with one exception. In the uninformed dual-cue condition, they were told about the associative relation but letter overlap was never mentioned. When cues were presented only during 
test, the instructions for study indicated that the items would be presented only once, that the subjects must concentrate, and so on.

Immediately following the last presentation, instructions for recall were read. The instructions either reminded subjects about the nature of the relationship of cues to targets, or, as when cues were presented only during test, they specified the nature of the relationship. Thus, when cues were shown at test only, information about retrieval cues was provided after the items were encoded. This procedure avoided biasing the subject toward encoding either sensory or semantic features prior to study. All instructions were equated for length. Recall was always oral.

Finally, in the uninformed dual-cue conditions, the subjects were read a carefully constructed set of questions designed to determine their degree of awareness of the first-letter sharing between cues and targets.

\section{Subjects}

Sixteen subjects were assigned to each condition, eight to each list. Thus, there were a total of 176 subjects in the entire experiment. All subjects were selected from courses in introductory psychology and received points toward their grade for participation.

\section{RESULTS}

Table 1 displays mean correct responses for location of cue and cuing conditions. A 2 by 5 analysis of variance of the data indicated that location $[F(1,150)=57.33]$, conditions $[F(4,150)=26.56]$, and the Location by Conditions interaction $[F(4,150)=5.58]$ were all significant $(\mathrm{p}<.01, \mathrm{MSe}=6.87)$. Fisher's two-tailed least significant difference (1.s.d.) for comparisons among the cue conditions was 1.30 . Thus, recall was generally better when cues were presented during study, and recall was highest with dual cues, next highest with associatively related words, and lowest with first-letter cues. The greater effectiveness of the dual cues was not dependent upon being informed about the first-letter overlap between cue and target. A separate 2 by 2 analysis of the dual-cue conditions indicated that location was reliable $[F(1,60)=68.81$, $p<.01]$, but that information and the interaction between these two sources were not $(F s=2.14$ and 1.55, MSe = 5.32). This result is interesting when

Table 1

Mean Correct Responses as a Function of Location of Cue and Condition

\begin{tabular}{lrr} 
& \multicolumn{2}{c}{ Location of Cue } \\
\cline { 2 - 3 } Condition & \multicolumn{3}{c}{$\begin{array}{c}\text { Study and } \\
\text { Test Only }\end{array}$} & Test \\
\hline First letter embedded in related word: & & \\
$\quad$ Uninformed (GLAMOUR GIRL) & 9.13 & 14.63 \\
Informed (GLAMOUR GIRL) & 10.69 & 14.75 \\
Related word only (DAINTY GIRL) & 7.81 & 12.31 \\
First letter only (G GIRL) & 8.75 & 9.75 \\
First letter embedded in unrelated word: & & \\
$\quad$ Informed (GHOSTLY GIRL) & 6.31 & 6.94 \\
Unrelated word only (ACCOUNT GIRL) & & 4.94 \\
\hline
\end{tabular}

considered along with the verbal report data. When subjects were not told about the first-letter contingency, 13 out of the 16 subjects in the test-only condition indicated that they never noticed this relationship. In the study-test condition, only half of the subjects became aware of the relationship, four during the initial portion of study and four during the middle portion of study. These findings suggested that awareness of the sensory contingency may not be a necessary requirement for dual-cue facilitation of recall.

In the overall analysis of variance, the reliable interaction between location and cuing condition (1.s.d. $=1.83$ ) indicated that presenting cues during study facilitated recall only in the dual-cue and in the related-word conditions, only when cues were associatively related to their targets. Recall was unaffected by cue availability during study when first letters served as cues, regardless of whether the cues were presented alone or embedded in unrelated words. A separate analysis of first-letter conditions $(\mathrm{MSe}=9.21)$ indicated that, although embedding first-letter cues reduced recall $[F(1,60)=11.97]$, location and the interaction between location and embedding were not significant (both Fs near unity). Thus, having the associative cues available during encoding facilitated subsequent retrieval. Having first-letter sensory cues available during study had no effect relative to having these cues only at test. These findings are consistent with those obtained with synonym and rhyme cues (Nelson et al., 1974).

Regardless of whether cues were located at test only or during both study and test phases, recall with dual cues reliably exceeded recall with single cues. Study-test conditions were analyzed separately to determine if the first-letter and associative cues had additive effects upon recall. The dual-cue condition (informed) combined with the related-word, first-letter embedded, and unrelated-word conditions form a 2 by 2 factorial, with the factors defined as presence/absence of associative cues and presence/absence of letter cues. First-letter embedded was deemed a more appropriate baseline as compared to the first-letter-only condition. With this control, every subject received words during study and during test. For convenience, mean correct responses for these conditions are displayed in the left-most two columns of Table 2 . The results of the statistical analysis indicated that the presence of associative cues and the presence of first-letter cues facilitated recall $\left[F_{s}(1,60)=118.14\right.$ and 10.08 , $\mathrm{MSe}=7.81]$. These facilitating effects were independent and additive. The interaction between cue types was less than unity. Thus, dual associative first-letter cues increased recall by about $61 \%$ (9.8 items), associative cues alone by $46 \%$ ( 7.4 items), and the first-letter cues alone by about $13 \%$ ( 2.0 items). Similar values were provided by subtracting recall in the single-cue conditions from dual-cue recall. However, recall in the dualcue conditions was very high and near the ceiling of 16 
Table 2

Mean Correct Responses as a Function of the Presence/Absence of Associative and Letter Cues and Study Rate

\begin{tabular}{cccccc}
\hline & \multicolumn{2}{c}{ Slow $(3.0 \mathrm{sec})$} & & \multicolumn{2}{c}{ Fast $(1.2 \mathrm{sec})$} \\
\cline { 2 - 3 } \cline { 5 - 6 } Letter Cues & \multicolumn{2}{c}{ Associative Cues } & & \multicolumn{2}{c}{ Associative Cues } \\
Present & Absent & & Present & Absent \\
\hline Present & 14.75 & 6.94 & & 12.87 & 4.19 \\
Absent & 12.31 & 4.94 & & 10.94 & 2.44 \\
\hline
\end{tabular}

items. The possibility remained that recall performance in this condition underestimated the real potential of the combined cues. To evaluate this possibility, the portion of the experiment including presence/absence of associative and of letter cues was replicated using a faster $1.2 \mathrm{sec}$ rate of study presentation. Mean correct recalls for each of these conditions are displayed in the right-most two columns of Table 2. Analysis of the combined experiments indicated that the presence of associative cues, letter cues, and the slower rate facilitated recall $\left[F_{s}(1,20)=274.88,17.28,18.91\right.$, MSe $=7.64]$. All effects were additive. None of the interactions between these three sources were reliable, with all Fs near unity. Thus, the faster rate reduced recall proportionally in all conditions by about two items. The additivity obtained in the initial experiment apparently was not caused by a ceiling effect in the dualcue conditions. Estimates of the relative effects of the cues pooled over the two experiments remained essentially unchanged.

Estimates of cue effectiveness for different cue types also were obtained with the conditions involving the presentation of cues only at test. The dual-cue and first-letter embedded comparison indicated that associative cues facilitated recall by $27 \%$ (4.4 items). The dual-cue and related-word comparison indicated that first-letter cues added about 18\% (2.9 items). Comparison of the values obtained for test only and study-test conditions confirmed the earlier conclusion. The effectiveness of associative cues was substantially improved, nearly doubled, when these cues were presented at study and again at test. By contrast, the effectiveness of first-letter cues remained unchanged by presenting them during study.

\section{DISCUSSION}

GLAMOUR is a better cue for retrieving the episodically encoded representation of GIRL than either DAINTY or GHOSTLY. Dual sensory-associative cues facilitated recall above and beyond the facilitation produced by the individual cues alone. This dual facilitation appeared to reflect the independent and additive contributions of the individual cues; it was observed when cues were shown only at test or during both study and test and, finally, the effect apparently did not depend upon the subjects' being explicitly aware of the additional sensory overlap contingency.
These results are consistent with the general assumption that information about appearance and significance is activated during encoding, and that each of these types of information can independently facilitate retrieval. As such, these results are consistent with the encoding and retrieval assumptions of the CAR formulation.

The assumptions of the CAR model also were bolstered by the finding that the influence of associative but not of sensory cues was highly affected by presenting these cues during study. The presence of the associative cue during study biases the encoding of cue and target toward activating specific and unique semantic features shared by these items (cf. Tulving \& Thomson, 1973). By comparison, if the target word is shown alone during study, the activation of these specific and unique shared features would be less likely. Thus, given access to the target by the cue during generation, the likelihood of recognizing the target should be greater when the cue was available during study, primarily because of the prior activation of differentiating common attributes. For example, if the target word GIRL is presented in the presence of the cue GLAMOUR, the meaning of GIRL as it related to physical attractiveness, modeling, and so on, is likely to be activated. If the target word GIRL is presented alone, some portion of the features related to the glamour meaning may be primed, but the activation of these specific meaning features would appear to be less likely. Hence, when GLAMOUR is presented during the test trial and GIRL is generated as an alternative, the probability of correctly recognizing it as a target word in the list would be greater if meaning features specifically related to glamour were activated during study.

Presenting the sensory cues during study had little additional effect relative to presenting them only during test. According to the model, the availability of sensory cues during encoding should bias the processing of the cue and target toward activating shared sensory features. However, in this case, the sensory cue is completely redundant with respect to its target, adding virtually no unique information beyond that obtained during normal processing or coding of the target. For example, the activation of the sensory codes related to the $\mathrm{G}$ in the target GIRL is not enhanced if GLAMOUR is available during encoding, not enhanced relative to processing GIRL without the cue. This analysis and the example suggests that the facilitating effects of the activated sensory features are primarily if not solely limited to the retrieval phase of the task. Thus, in accordance with the CAR assumptions, adding a sensory contingency aids recall in either generation or recognition or in both of these phases. As a generation effect, the subjects may generate alternatives sharing both associative and first-letter relationships with the cue word. This restriction in plausible alternatives essentially would reduce the size of the set of relevant possibilities, an effect that should be reflected in higher recall, since 
there would be a smaller pool of items from which the critical one would be selected. Alternatively, the sensory contingency may have facilitated recall during the final phase of retrieval, during recognition. This process presumably involves a sampling of the features of each generated alternative to determine if they were activated during encoding. Hence, if the cue recapitulates or reactivates features shared with the target, whether they are sensory or semantic, recognition of activated features is enhanced, discrimination of list membership is facilitated and final recall is increased. At this point a choice between these two alternatives cannot be made on either logical or empirical grounds. All that can be said with reasonable assurance is that the facilitating effect of the additional sensory cue seems to be limited to retrieval processes. Accordingly, GLAMOUR is a better cue for recalling the word GIRL than is DAINTY or GHOSTLY because it places greater restrictions on possible target alternatives, because it independently reactivates both sensory and associative features during the process of determining list membership, or because it allows both of these processes to occur.

\section{REFERENCES}

BaHrick, H. P. A two-phase model for prompted recall. Psychological Review, 1970, 77, 215-222.

Craik, F. I., \& Lockrart. R. S. Levels of processing: A framework for memory research. Journal of Verbal Learring and Verbal Behavior, 1972, 11, 671-684.

Craik, F. I., \& Tulving, E. Depth of processing and the retention of words in episodic memory. Joumal of Experimental Psychology: General, 1975, 104, 268-294.

Nelson, D. L., WheEler, J. W., Borden, R. C., \& Brooks, D. H. Levels of processing and cuing: Sensory versus meaning features. Joumal of Experimental Psychology, 1974, 103, 971-977.

Shapiro, S. 1., \& Palermo, D. S. An atlas of normative free association data. Psychonomic Monograph Supplement, 1965, 2(Whole No. 28), 219-250.

ThORNDIKE, E. L., \& LoRge, I. The teacher's handbook of 30,000 words. New York: Bureau of Publications, Teachers College, Columbia University, 1944.

Tulving, E., \& Thomson, D. M. Encoding specificity and retrieval processes in episodic memory. Psychological Review, 1973, 80, 352-373.

(Received for publication December 17, 1976; revision accepted February 10, 1977.) 